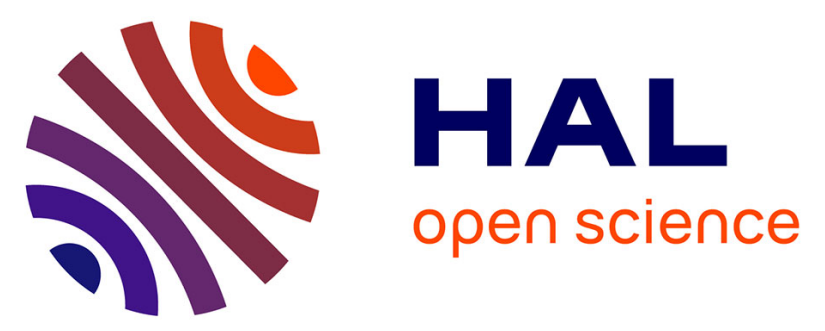

\title{
Use of Wavelet transform in the Study of Propagation of Transient Acoustic Signals Across a Plane Interface Between Two Homogeneous Media
}

Ginette Saracco, Alexandre Grossmann, Philippe Tchamitchian

\section{- To cite this version:}

Ginette Saracco, Alexandre Grossmann, Philippe Tchamitchian. Use of Wavelet transform in the Study of Propagation of Transient Acoustic Signals Across a Plane Interface Between Two Homogeneous Media. Wavelets, Wavelets, Time-Frequency Methods and Phase Space, 1ere Int. Wavelets Conf. Dec. 14-18, 1987, CIRM-Marseille, Springer Berlin Heidelberg, pp.139-146, 1989, inverse problems and theoretical imaging, 10.1007/978-3-642-75988-8_9 . hal-00549902

\section{HAL Id: hal-00549902 https://hal.science/hal-00549902}

Submitted on 6 Oct 2021

HAL is a multi-disciplinary open access archive for the deposit and dissemination of scientific research documents, whether they are published or not. The documents may come from teaching and research institutions in France or abroad, or from public or private research centers.
L'archive ouverte pluridisciplinaire HAL, est destinée au dépôt et à la diffusion de documents scientifiques de niveau recherche, publiés ou non, émanant des établissements d'enseignement et de recherche français ou étrangers, des laboratoires publics ou privés. 
Wavelets Time-frequency Methods and Phase Space

$1^{\text {st }}$ Int. Wav. Conf, Dec 14-18 1987, CIRM-Marseille

Edt Combes JM, Grosmmann A., Tchamitchian Ph.

SPRINGER-VERLAG, p. 139-146, 1989

\section{Use of Wavelet Transforms in the Study of Propagation of Transient Acoustic Signals Across a Plane Interface Between Two Homogeneous Media}

1Laboratoire de Mécanique et d'Acoustique, Equipe Ultrasons, C.N.R.S., 31, Chemin J. Aiguier, F-13402 Marseille Cedex 09, France

${ }^{2}$ Centre de Physique Thérique, Section II, C.N.R.S.

Luminy Case 907, F-13288 Marseille Cedex 09, France

${ }^{3}$ C.P.T. et Faculté des Sciences et Techniques de

Saint-Gerôme, F-13397 Marseille Cedex 13, France

\section{Introduction.}

The problem we study can be defined as follows: In three-dimensional space, we consider two homogeneous media - "air" and "water" - separated by plane interface. There is a point source of sound in "alr" at height $h$ above the interface. its emission is given by a function $F(t)$ of $t$ lme. We are interested in the behavlour of pressure in water, at time $t$, depth $z$, and distance $r$ from a vertical line going through the source.

This problem has been discussed Innumerable times, both for monochromatic and translent sources [1-6]. The feature of interest to us is the existence, first recognized in the monochromatic reglme, of contributions to the total solution called lateral waves. They tend to be concentrated near the Interface, and have propertles of propagation and attenuation different from waves in homogeneous media. They decrease exponentlally with depth, and thelr attenuation length is frequency-dependent. Consequently, their penetration depth decreases with frequency. For a recent discussion, see e.g. [7]. It should be mentloned that this attenuation does not correspond to absorption of energy; a lateral wave is merely a contribution to an elastic propagatlon.

In the case of time-limited sources, one can see the contributions of lateral waves arriving at times different from the "geometric" contrlbutions. At sufflclently shallow depths, they can contribute slgnificantly to the the total acoustlc field.

The classical methods of resolution, while well adapted to the case of monochromatic sources, are less sulted to the description of transients. This is due to the fact that waves of different trequencies both follow dlfferent paths and undergo different attenuations. In such time-and-irequency dependent situations, it Is natural to apply. wavelet transform technlques. As a matter of fact, the main motivation for the introduction of wavelet methods in J. Morlet's work was the need, in geophyslcs, to study frequency-dependent propagatlon phenomena.

In the first part of thls paper we shall brlefly describe the tlme behavlour of the wavelet transform of the acoustlc field at a fixed polnt under water. (This is not the same as attempting to use wavelet techniques to solve the partial differentlal equations of the propagation problem). It is straightforward to write down an expression for the wavelet transform of the propagator for our problem. This expression is much smoother than the propagator lisself, and allows selectlve reconstitutions with arbitrary precision. It has a natural decomposition into three contributions corresponding to branch-points of the Integrand. 
natural decomposition into three contributions corresponding to branch-points of the integrand.

These contributions, which we study separately, are obtained without the help of stationary phase approximations which are used in the standard definition of lateral waves. Nevertheless, the main features of the geometric and lateral waves can be seen in our decomposition. In order to avoid possible confusions we enclose our names in quotation marks. The expressions, given below, have been numerically evaluated. The results are discussed and displayed graphically.

In a second part, we use the different contributions to obtain a formula for reconstruction of the time-dependent source.

\section{Expressions for the propagator.}

All quantities of interest can be calculated from the scalar acoustic potential $\Phi(r, z, t)$. In the case described above, its Fourier transform with respect to time is given by the integral (1) below, where the notations are as follows:

$c_{1}$ : sound velocity in air; $n$ : the refraction index for water: one has $n<1$.

$m$ : ratio of densities of the two media: one has $m>1$.

$f(\omega)=\frac{1}{\sqrt{2 \pi}} \int e^{i \omega t} F(t) d t ; J_{0}:$ Bessel function.

(1)

$\hat{\Phi}(r, z, \omega)=\frac{i \operatorname{sgn}(\omega)}{c_{1}} \sqrt{\frac{2}{\pi}} \hat{F}(\omega)|\omega| \int_{0}^{\infty} J_{0}\left(\frac{u \omega r}{c_{1}}\right) N(\omega, u, z) \frac{1}{D(u)} u d u$,

where $N(\omega, u, z)=\exp \left\{i \operatorname{sgn}(\omega) \frac{|\omega|}{c_{1}}\left[h \sqrt{1-u^{2}}+z \sqrt{n^{2}-u^{2}}\right]\right\}$

and $D(u)=m \sqrt{1-u^{2}}+\sqrt{n^{2}-u^{2}}$.

Here, for $u>n$, the expression $\sqrt{n^{2}-u^{2}}$ is defined to mean $\mid \operatorname{sgn}(\omega) \sqrt{\left|\left(n^{2}-u^{2}\right)\right|}$.

Similarly, for $u>1$, we define $\sqrt{1-u^{2}}$ as $i \operatorname{sgn}(\omega) \sqrt{\left|\left(1-u^{2}\right)\right|}$.

We shall divide the integral over $u$ into three contributions, corresponding to the branch-points in the integrand:

$$
\Phi=\Phi_{1}+\Phi_{2}+\Phi_{3}
$$

The first contribution, which we call the "geometric", is defined by (1) where the integral over $u$ goes from 0 to $n$. The second, or "lateral" contribution corresponds to $n<u<1$; the last one, "evanescent", corresponds to $u>1$. These names have been chosen by analogy with the terminology used in the case of the monochromatic source.

3. Wavelet transform of the propagator: formulas.

In order to study the behaviour of the three components of $\Phi$, we introduce their continuous wavelet transform with respect to an analyzing wavelet $\mathrm{g}(\mathrm{t})$, which we assume progressive (i.e. without Fourier components for negative $\omega$; i.e. $g(t)$ is what is known as an analytic signal). With the conventions of the present paper, the wavelet transform of each of the three components (2) is given by: 


$$
(L \Phi)(b, a)=\sqrt{a} \int_{0}^{\infty} \hat{\Phi}_{j}(r, z, \omega) \hat{g}(a \omega) e^{-i b \omega} d \omega .\langle j=1 . .3\rangle
$$

We have evaluated these expressions in the case where $\hat{F}(\omega)=$ const, i.e. where the source emits a $\delta$-function pulse. The analyzing wavelet, in frequency space, was chosen as $\hat{g}(\omega)=\exp \left(-\frac{(\omega-5.5)^{2}}{2}\right)$; this is the "standard Morlet wavelet" which has been extensively used (See e.g. [8]). For all practical purposes, it is admissible and progressive. In our analytic and numerical evaluation, we do not make any use of asymptotic estimates.

4. Wavelet transform of the propagator: numerical results.

In the numerical evaluation of the propagator and of its wavelet transform, we have used algorithms of the Romberg type, adapted to the somewhat singular behaviour of the integrand at the boundary.

In all the graphic representation, it is the level lines that are displayed. The modulus of the transform is shown in logaritmic scale, with a dynamic speen of $40 \mathrm{db}$. The phase represeritation is linear, between $-\pi$ and $\pi$. The color scale is in increasing order : mauve, blue, green, yellow, orange, red and black. For phases, mauve has been replaced by red, for the sal of better visualization.

The values of $m, n$ chosen for the computation are respectively 800 and 0.2266

The figures $1 p$ ( $p$ for $p h a s e$ ) and $1 m$ ( $m$ for modulus) correspond to an observation point directly under the source (i.e $r=0$ ), at a finite depth $z$ under the interface $(z / h=0.2$ ). The functions displayed are the phase and the modulus, respectively, of the wavelet transform of the contribution $\Phi_{1}$, considered as a function of time. The moment of arrival of this contribution can be seen as the vertical line of constant phase on Fig 1p, corresponding to the abscissa a little over 3.0. The same abscissa appears as a line of maximum modulus on any horizontal line on Figure $1 \mathrm{~m}$. This result is a very clear indication of the arrival of a discontinuity. Quantitatively, the position of this discontinuity corresponds exactly to the calculated time of arrival.

In figures $2 \mathrm{~m}$ and $3 \mathrm{~m}$ the point of observation is at a finite radial distance from the source (namely $r / h=1)$, and at a shallow depth $(z / h=0.1)$. In figure $2 m$, one sees the modulus of the wavelet transform of the "geometric" contribution $\Phi_{1}$. The figure $3 \mathrm{~m}$, which uses exactly the same coordinate system and graphical conventions as $2 \mathrm{~m}$, displays the modulus of the wavelet transform of the "lateral" contribution $\Phi_{2}$. It is very instructive to compare the two pictures. One notices that the "geometric" contribution arrives before the "surface contribution", in agreement with theory. The figure $4 \mathrm{~m}$ describes the "lateral" contribution at $r / h=2$.

We have also calculated, independently, the contributions to the propagator. The results, for the modulus of "lateral" contribution, are shown in Figs. 5 and 6 for different positions of the observation point.

A more systematic and quantitative discussion is in preparation [9]. 

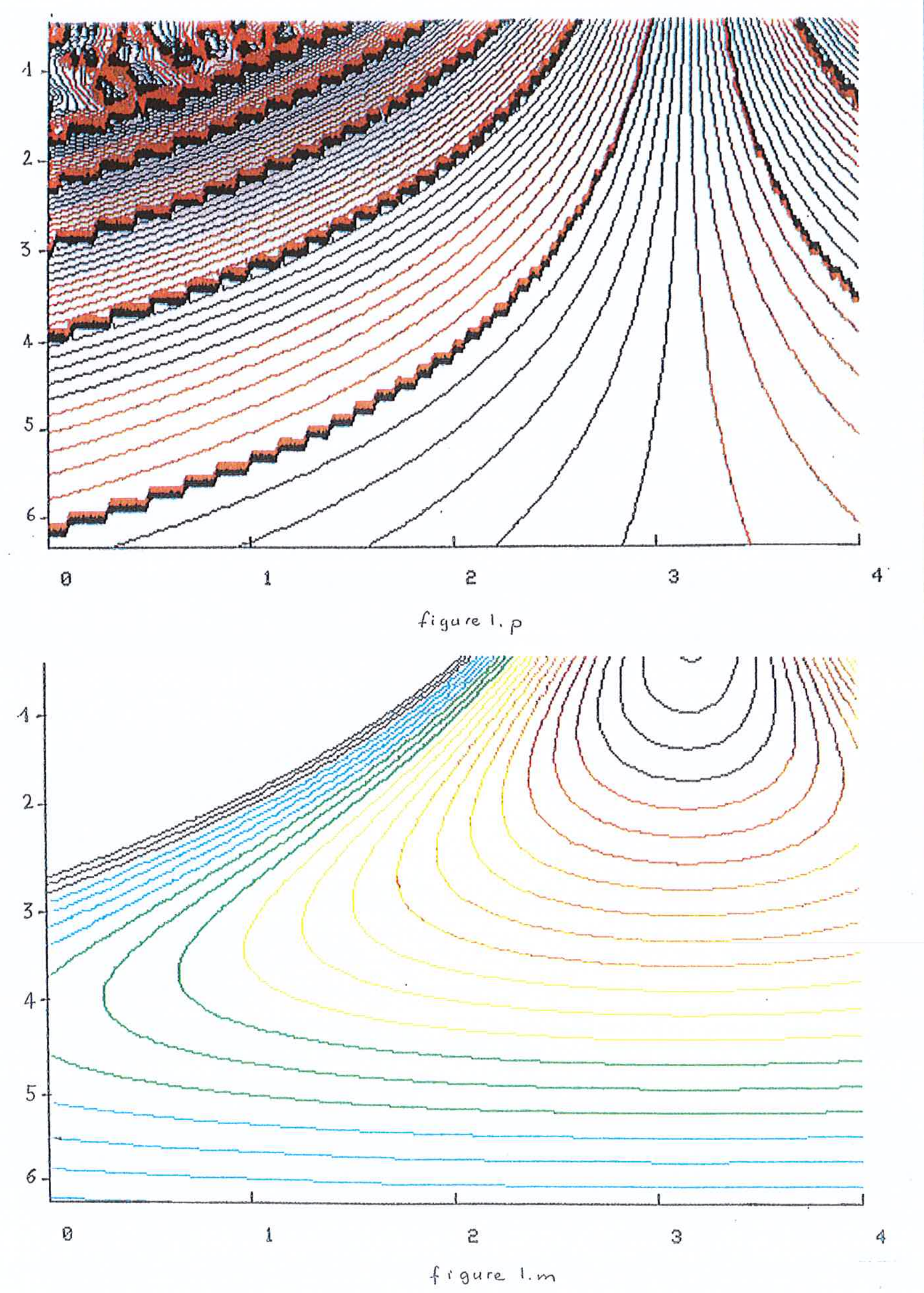


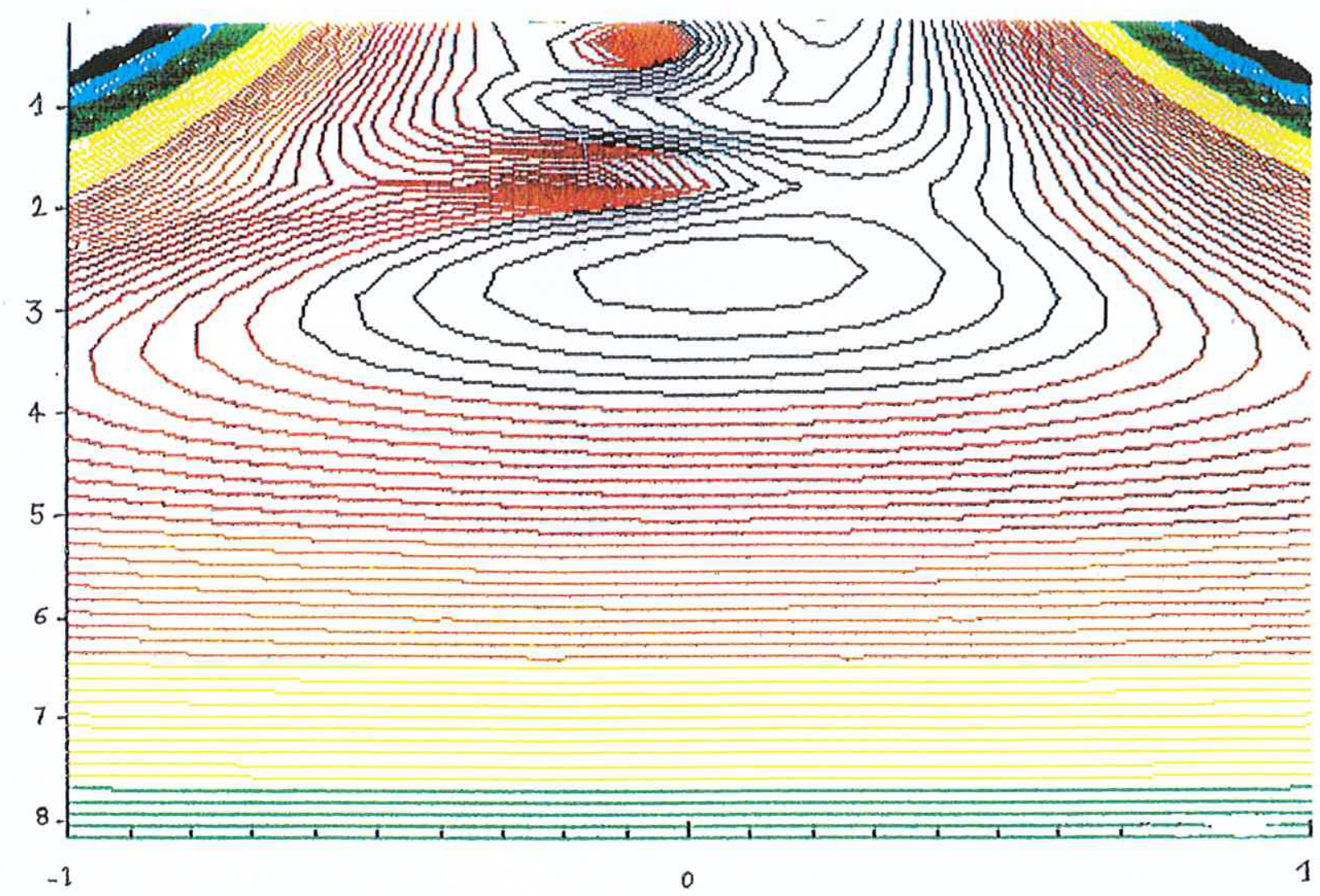

figure $2 . \mathrm{m}$

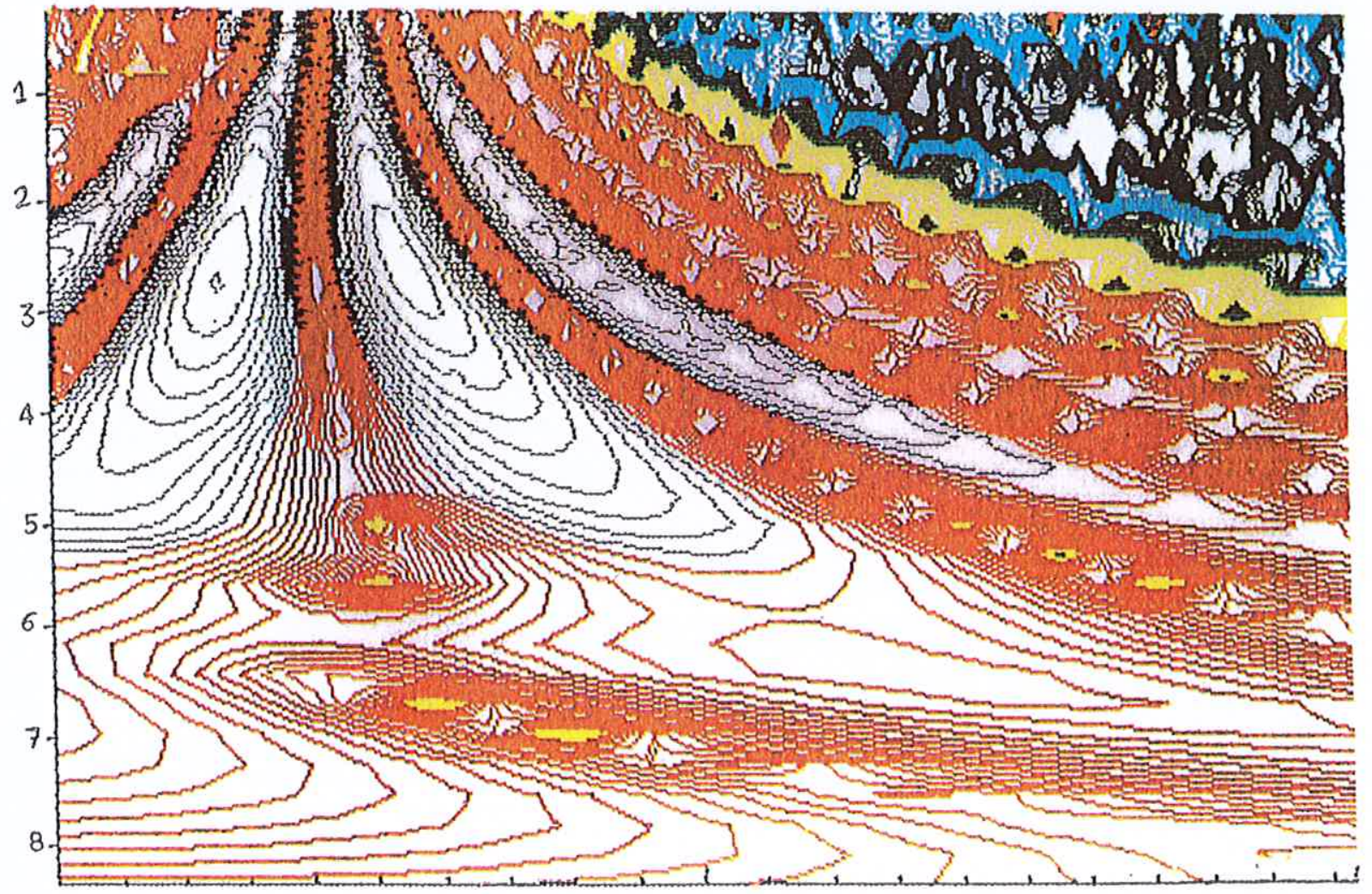

$\theta$ 


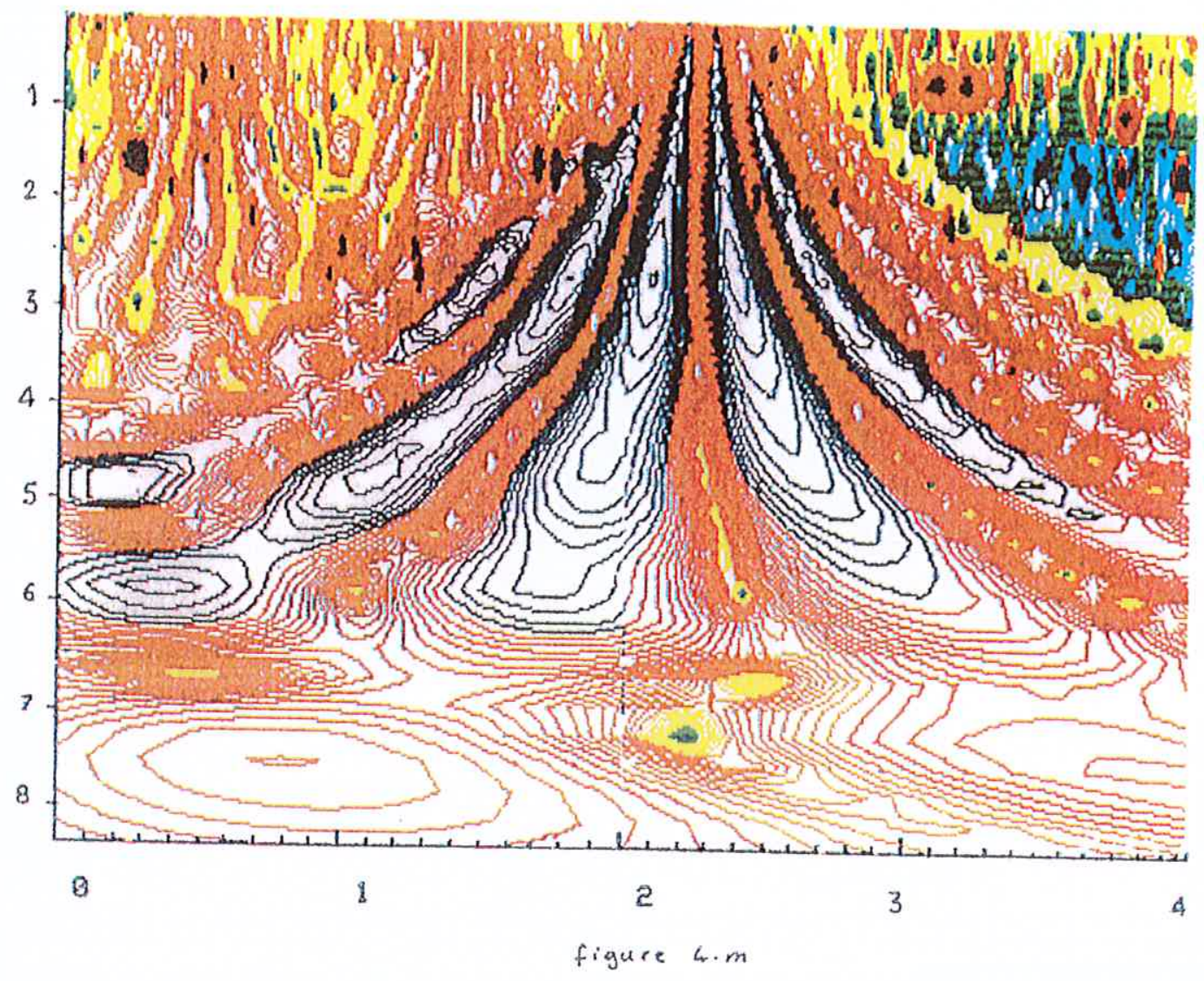

Figure 1p: Phase of the wavelet transform of the "geometric" contribution. The observation point is under the source, $r / h=0$ at a depth of $z / h=0.2$. The smallest dilation parameter is equal to 0.3 . The number of voices per ootave is 5 in a range of 5 octaves.

Figure $\mathrm{im}$ : Modulus of the wavelet transform of the "geometric" contribution with the same parameters of the figure $1 \mathrm{p}$.

Figure $2 \mathrm{~m}$ : Modulus of the wavelet transform of the "geometric" contribution at a radial distance from the source $\mathrm{r} / \mathrm{h}=$ 1 and a shallow depth of $z / h=0.1$. Here, the smallest dilation parameter has been taken equal to 0.15 , with 5 voices per octave. The number of octaves is 6 .

Figure $3 \mathrm{~m}$ : Modulus of the wavelet transform of the "lateral" contribution at the observation point of the "geometric" contribution seen in figure $2 \mathrm{~m}$. The analysis of the signal was performed with the same scale parameters and the same graphical conventions as in figure $2 \mathrm{~m}$.

Figure $4 \mathrm{~m}$ : Modulus of the wavelet transform of the lateral contribution where the radial distance is $\mathrm{r} / \mathrm{h}=2$. The signal was analyzed with scale parameters equal to those of figure $2 \mathrm{~m}$. 


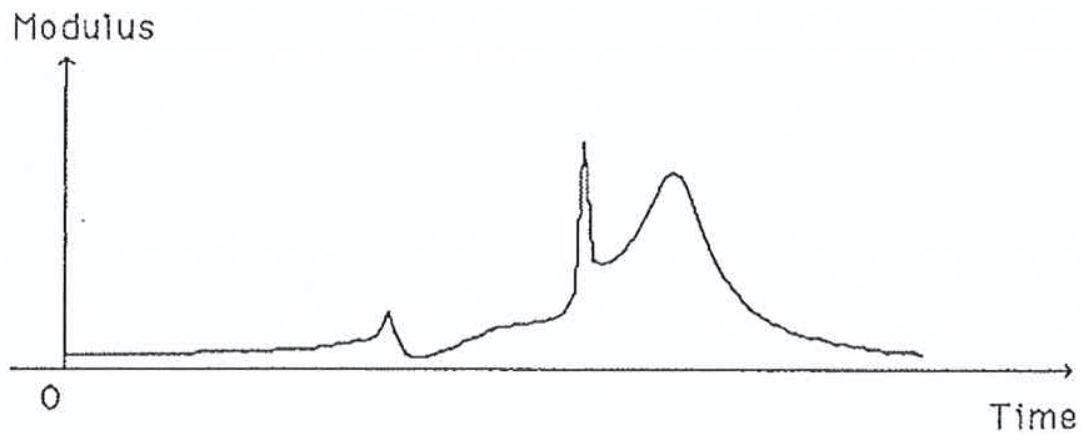

ta

$t_{1} t_{2} t_{1}$

Figure 5

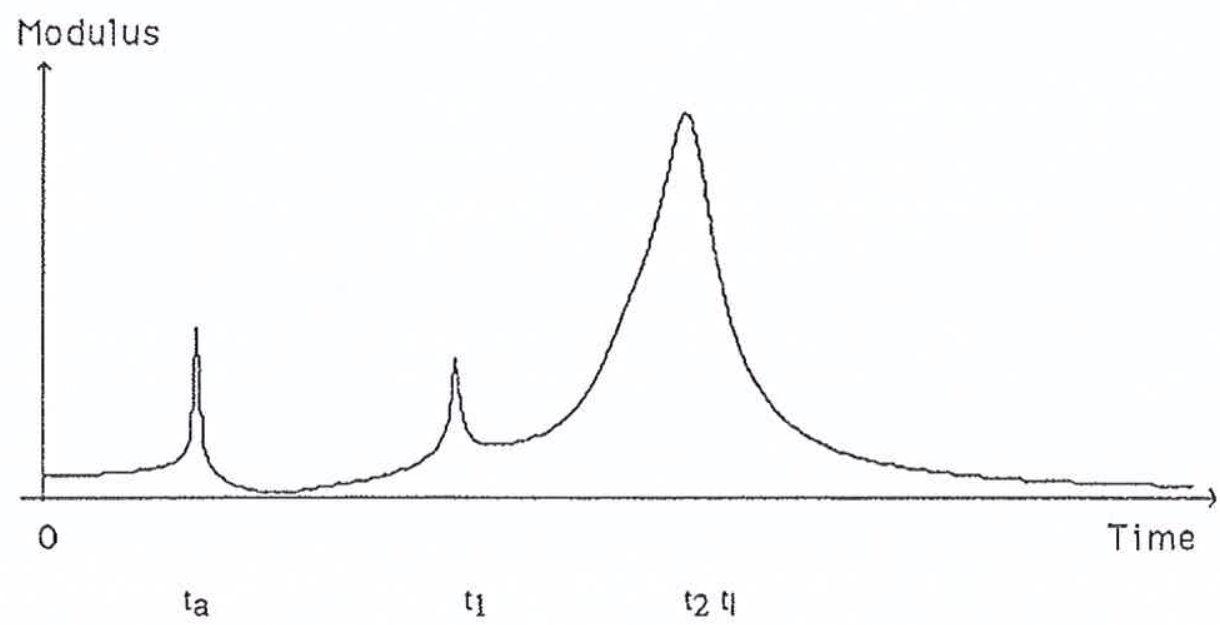

Figure 6

Figures 5, 6: Modulus of the contribution of lateral waves to the propagator (Green'sfunction) for $r / h=1$ and $r / h=2$ respectively.

$\mathrm{t}_{\mathrm{a}}=$ artefact due to the decomposition into geometric and lateral contribution seen [9] for a detailed explanation.

$\mathrm{t}_{1}, \mathrm{t}_{2}=$ delays appearing in formula (4).

$y=$ time of arrival of the "lateral" wave.

5. A reconstruction formula.

We now return to the consideration of a point source with arbitrary time variation $F(t)$. Our aim is to reconstruct $F(t)$ from information obtained through "underwater listening". It turns out that there exists a simple reconstruction formula that involves the three contributions, evaluated at all depths for a fixed horizontal distance $r$. The notations being as above, define $P(r, z, t)$ as the pressure obtained from the total acoustic potential $\Phi$. The formula to be written is valid asympotically as $r / h \rightarrow \infty$. Consider $l(r, t)=\int P\langle r, z, t\rangle d z$.

$$
\text { Define } t_{2}=\frac{\sqrt{r^{2}+h^{2}}}{c_{1}} \text { and } t_{1}=\frac{h \sqrt{1-n^{2}}}{c_{1}}+\frac{r}{c_{2}} \text {. }
$$

We have then:

(4) $I(r, t) \approx C(r, h) F\left(t-t_{1}\right)+K(r, h) F\left(t-t_{2}\right)$ 
where $C(r, h)$ and $K(r, h)$ can be explicitly calculated. If $t_{2}-t_{1}$ is larger than the duration of the emitted sound $F(t)$, we obtain the exact signal emitted in air repeated twice but with different amplitudes. We have not yet performed any numerical evaluations of (4).

Acknowlegdements: One of the authors (S. G.) thanks Dr C. Gazanhes for helpful advice. This work has been partially supported by the company DIGILOG.

References.

[1] Gerjuoy, E., Refraction of Waves from a point source into a Medium of Higher Velocity, Phys. Rev. 73 (1948) 1442-1449.

[2] Brekhovskikh, L. N., Waves in layered Media (Wiley, New York) 1960, pp. 292-302.

[3] Gottlieb, P., Sound Source near a Velocity Discontinuity, J. Acoust. Soc. Am. 32 (1960) $1117-1122$.

[4] Towne, D. H., Pulse shapes of Spherical Waves Reflected and Refracted at a Plane Interface separating Two homogeneous Fluids, J. Acoust. Soc. Am. 44 (1968) 65-76.

[5] De Hoop, A. T. and Van Der Hijden, J., Generation of Acoustic Waves by an Impulse point Source in a fluid/solid configuration with a Plane boundary, J. Acoust. Soc. Am. $\underline{7} 5$ (1984) 1709-1715.

[6] Casserau, D., Nouvelles Méthodes et Applications de la Propagation Transitoire dans les milieux Fluides et Solides, Thèse de I'U. E. R. Paris VII (1988).

[7] Saracco G., Transmission acoustique à travers le dioptre air-eau, J. Acoust. (1988) 171 79.

[8] Grossmann, A. and Morlet, J., Decomposition of Hardy functions into square integrable wavelets of constant shape. SIAM Journ. of Math. Analysis, 15(1984) 723-736.

[9] Grossmann, A., Saracco, G., Tchamitchian, P., Study of propagation of transient acoustic signals across a plane interface with the help of the wavelet transform, in preparation. 